\title{
População em situação de rua: perfil dos casos de coinfecção tuberculose e HIV
}

\section{Homeless people: profile of tuberculosis and HIV coinfection cases}

\section{Nathalia Halax Orfão' \\ Kamila Maria da Silva ${ }^{2}$ \\ Melisane Regina Lima Ferreira ${ }^{3}$ (C) Maria Eugenia Firmino Brunello 4 (1)}

\begin{abstract}
${ }^{1}$ Autora para correspondência. Universidade Federal de Rondônia (Porto Velho). Rondônia, Brasil. nathaliahalax@unir.br
\end{abstract}
2-3Universidade Federal de Rondônia (Porto Velho). Rondônia, Brasil. kamila.m.silva00@gmail.com, melisane1206@gmail.com

${ }^{4}$ Faculdade de Tecnologia em Saúde (Ribeirão Preto). São Paulo, Brasil. maria.brunello81@gmail.com

RESUMO | OBJETIVO: Analisar o perfil da População em Situação de Rua (PSR) com a coinfecção tuberculose (TB)/HIV em Porto Velho-RO, entre 2015 a 2018. MATERIAIS E MÉTODOS: Estudo descritivo, transversal com abordagem quantitativa realizado a partir do levantamento das variáveis sociodemográficas, clínicas e do tratamento dos casos no Sistema de Informação Nacional de Agravos de Notificação, e posteriormente análise por distribuição de frequência, após atender aos preceitos éticos. RESULTADOS: Foram notificados sete casos de TB/ HIV entre as PSR. A maioria era do sexo feminino, entre 31 a 38 anos, parda, baixa escolaridade, apresentavam doenças e agravos associados, não eram população especial, notificadas em 2016, na referência terciária, era caso novo ou reingresso após abandono, forma clínica pulmonar, radiografia de tórax suspeito, baciloscopia de escarro negativa, teste molecular rápido para a TB e cultura não realizadas, teste de sensibilidade e controle mensal em branco, até três contatos identificados e nenhum examinado, não realizaram Tratamento Diretamente Observado, tratamento na referência terciária, de 61 a 120 dias e mais do que 181 dias, e elevado percentual de abandono. CONCLUSÃo: Torna-se essencial rever as estratégias de acesso aos serviços e ações ofertadas para atender as demandas de saúde da PSR, voltadas para ambos os agravos.

DESCRITORES: Pessoas em Situação de Rua. Tuberculose. HIV. Coinfecção.
ABSTRACT | OBJECTIVE: To analyze the Homeless Population profile with tuberculosis (TB)/HIV coinfection in Porto Velho - RO, between 2015 and 2018. MATERIALS AND METHODS: A descriptive, cross-sectional study with a quantitative approach carried out from the survey the sociodemographic, clinical variables and the treatment of cases in the National Information System on Notifiable Diseases, and later analysis by frequency distribution, after meeting the ethical precepts. RESULTS: Seven cases of TB/HIV were reported as homeless population. The sample was predominantly female, between 31 and 38 years old, mulattos ("pardos"), low schooling, had associated diseases and illnesses, were not special segments, notified in 2016, in the tertiary reference, new cases or reentry after abandonment, pulmonary clinical form, suspected chest $X$-ray, negative sputum smear microscopy, molecular rapid test for TB and culture not performed, sensitivity test and blank monthly control, up to three identified contacts, and none examined, did not perform Directly Observed Treatment, were treated in the tertiary referral, from 61 to 120 days and more than 181 days, and a high percentage of abandonment. CONCLUSION: It is essential to review the strategies for accessing services and actions offered to meet the health demands of the Homeless Population, aimed at both diseases.

DESCRIPTORS: Homeless Persons. Tuberculosis. HIV. Coinfection. 


\section{Introdução}

A Tuberculose (TB) é um problema de saúde pública vigente e pertinaz, ocasionando, no ano de 2018 , um total de 10 milhões de casos novos e aproximadamente 1.451 milhão de óbitos. O Brasil está entre os países com maior carga da doença no mundo, sendo um dos prioritários para a coinfecção tuberculose/ Vírus da Imunodeficiência Humana (TB/HIV), de acordo com a Organização Mundial da Saúde (OMS)ํㅗ.

No Brasil, estimou-se que, em 2018, tenham sido notificados 72.788 casos novos de TB (coeficiente de incidência de 34,8 casos $/ 100$ mil hab.), dos quais $8,8 \%$ apresentavam coinfecção TB/HIV, além de 4.490 óbitos (coeficiente de mortalidade de 2,2 óbitos/100 mil hab). No mesmo período, Rondônia registrou um coeficiente de incidência de TB de 28,5 casos $/ 100$ mil hab. e mortalidade de 1,3 casos/100 mil hab., ainda que a maioria destes casos $(59,5 \%)$ tenha ocorrido na capital - Porto Velho, que possui tais coeficientes superiores ao estado, tais como 59,5 casos/ 100 mil hab. e 1,5 óbitos/100 mil hab., respectivamente ${ }^{2,3}$. De modo complementar, $72,2 \%$ dos casos novos de TB foram testados para o HIV no estado e 7,7\% eram coinfectados TB/HIV $\underline{\text { }}$.

A População em Situação de Rua (PSR) é caracterizada pelas condições de vulnerabilidade, tais como: extrema miséria, exposição à violência, restrição aos bens básicos de sobrevivência, fragilidade psicossocial, exclusão social, frequente migração, bem como a elevada relação com álcool e drogas. Dados epidemiológicos mostram que o risco de adoecimento por TB entre essa população é 56 vezes maior quando comparado à população em geral ${ }^{4}$. Essa gama de fatores acaba dificultando o acompanhamento dos casos, controle de contatos, bem como a realização do Tratamento Diretamente Observado (TDO) para a TB 4,5 .

Os principais obstáculos encontrados pelas PSR no enfrentamento da TB estão relacionados à dificuldade no acesso aos serviços de saúde, retardo no diagnóstico e início do tratamento, à fragilidade de políticas intersetoriais, comunicação e articulação para integração horizontal e vertical ${ }^{6}$.

Ao mesmo tempo, a associação entre PSR e a coinfecção TB/HIV configura-se como um fenômeno multicausal, na qual diante do diagnóstico da TB, é preconizado o rastreamento do HIV nas unidades de saúde por meio do teste rápido, além do monitoramento anual da TB entre as Pessoas Vivendo com HIV (PVHIV), considerando que o comprometimento do sistema imunológico eleva o risco de adoecimento, e a TB por se caracterizar como uma doença oportunista, é a que mais leva estas populações ao óbito4.

Neste sentido, este estudo buscou analisar o perfil das pessoas vivendo em situação de rua coinfectadas com TB/HIV em Porto Velho-RO, no período de 2015 a 2018.

\section{Materiais e métodos}

Trata-se de um estudo descritivo, realizado de forma transversal, utilizando abordagem quantitativa, desenvolvido no município de Porto Velho, capital do estado de Rondônia, pertencente à região amazônica, que faz fronteira com a Bolívia e com os estados brasileiros do Amazonas, Mato Grosso e Acre. O município possui 34.090,962 km² em área territorial, população estimada, em 2018, de 519.531 habitantes, indicando uma densidade demográfica de 12,57 habitantes $/ \mathrm{km}^{2}{ }^{2}$.

A atenção à TB no município possui como porta de entrada a Atenção Primária à Saúde (APS), tendo como principais competências a busca por Sintomáticos Respiratórios (SR), solicitação de exames diagnósticos para a TB e HIV, notificação no Sistema de Informação de Agravos de Notificação (SINAN), acompanhamento do tratamento, oferta do TDO, avaliação dos contatos e, se necessário, encaminhamento para os outros níveis de atenção. 
De modo complementar, o município conta com a rede de apoio a nível ambulatorial para o acompanhamento de TB infantil, forma clínica extrapulmonar e coinfecção TB/HIV; além do nível terciário que se constitui como referência estadual para os casos de TB drogarresistente (TB-DR), Micobactéria Não Tuberculosa (MNT) e internação por complicações, se necessário. Como suporte laboratorial, conta com um a nível municipal para a realização da baciloscopia de escarro e Teste Molecular Rápido para a TB (TMR-TB), além de outro laboratório a nível estadual, responsável pela cultura de escarro e teste de sensibilidade.

Atualmente, a APS totaliza 54 unidades de saúde, divididas em 17 Unidades Básicas de Saúde (UBS) e 37 Unidades de Saúde da Família (USF), nas quais 20 estão localizadas na zona urbana e 33 na área rural, sendo subdivididas em 18 unidades terrestres e 15 ribeirinhas, bem como uma unidade móvel fluvial, contando ainda com uma Equipe de Consultório na Rua para atendimento da área urbana ${ }^{8}$.

A população do estudo foi constituída por todos os registros dos casos de TB entre a PSR notificados em Porto Velho, no SINAN, no período de 2015 a 2018. Como critérios de inclusão, foram considerados os registros dos casos de coinfecção TB/HIV, e como exclusão, aqueles cujos desfechos do tratamento estavam em branco.

A coleta de dados foi realizada a partir do levantamento das variáveis sociodemográficas (sexo, faixa etária, raça/cor, escolaridade, doenças e agravos associados e população especial), clínicas (ano da notificação, unidade de notificação, tipo de caso, forma clínica, exames diagnósticos (radiografia de tórax, baciloscopia de escarro, TMR-TB, cultura de escarro e teste de sensibilidade), HIV e de controle mensal (baciloscopia de escarro)), e do tratamento (controle de contatos, unidade de tratamento, regime de tratamento do TDO, data de início e término do tratamento, e situação de encerramento) no SINAN.

Os dados coletados foram armazenados no programa Excel da Microsoft, e posteriormente analisados a partir do software Statistica 13.4, da TIBCO, por meio de distribuição de frequência.

Atendendo às recomendações da resolução 466/12 do Conselho Nacional de Saúde ${ }^{9}$, o projeto matriz intitulado "Avaliação da situação epidemiológica da tuberculose no município de Porto Velho - RO" foi aprovado pelo Comitê de Ética em Pesquisa da Fundação Universidade Federal de Rondônia, conforme parecer n' 2.399.327.

\section{Resultados}

No período de 2015 a 2018, foram notificados 3.050 casos de TB em Rondônia, dos quais 2.050 (67,2\%) foram em Porto Velho, sendo 48 casos $(2,3 \%)$ entre a PSR, e destes, 7 casos (14,6\%) possuíam a coinfecção TB/HIV.

Em relação ao perfil sociodemográfico, a maioria pertencia ao sexo feminino $(57,1 \%)$, com uma faixa etária entre 31 a 38 anos em ambos os sexos $(71,4 \%)$, raça/cor autodeclarada parda $(85,7 \%)$, de um a quatro anos de estudo (42,9\%). Apresentavam doenças e agravos associados, tais como aids (100\%), alcoolismo $(57,1 \%)$, uso de drogas ilícitas (100\%), e não se caracterizavam como população especial (Tabela 1). 
Tabela 1. Distribuição do perfil sociodemográfico das pessoas em situação de rua coinfectadas TB/HIV, quanto ao sexo feminino e masculino em Porto Velho, Rondônia, no período de 2015 a 2018

\begin{tabular}{|c|c|c|c|c|c|c|c|}
\hline \multirow{2}{*}{ Variáveis } & & \multicolumn{2}{|c|}{ Feminino } & \multicolumn{2}{|c|}{ Masculino } & \multicolumn{2}{|c|}{ Total } \\
\hline & & $\mathbf{n}$ & $\%$ & $\mathbf{n}$ & $\%$ & $\mathbf{n}$ & $\%$ \\
\hline \multicolumn{8}{|l|}{ Faixa etária (anos) } \\
\hline 31 a 38 & & 2 & 50 & 3 & 100 & 5 & 71,4 \\
\hline 39 ou mais & & 2 & 50 & - & - & 2 & 28,6 \\
\hline \multicolumn{8}{|l|}{ Raça/cor } \\
\hline Parda & & 3 & 75 & 3 & 100 & 6 & 85,7 \\
\hline Preta & & 1 & 25 & - & - & 1 & 14,3 \\
\hline \multicolumn{8}{|l|}{ Escolaridade } \\
\hline 1 a 4 anos & & 2 & 50 & 1 & 33,3 & 3 & 42,9 \\
\hline 5 a 8 anos & & 2 & 50 & - & - & 2 & 28,6 \\
\hline Mais do que 8 anos & & - & - & 2 & 66,7 & 2 & 28,6 \\
\hline \multicolumn{8}{|l|}{ Doenças e agravos associados* } \\
\hline Aids & Sim & 4 & 100 & 3 & 100 & 7 & 100 \\
\hline \multirow{2}{*}{ Alcoolismo } & $\operatorname{Sim}$ & 2 & 50 & 2 & 66,7 & 4 & 57,1 \\
\hline & Não & 2 & 50 & 1 & 33,3 & 3 & 42,9 \\
\hline Uso de drogas ilícitas & $\operatorname{Sim}$ & 4 & 100 & 3 & 100 & 7 & 100 \\
\hline \multirow{2}{*}{ Tabagismo } & Não & 4 & $100-$ & 2 & 66,7 & 6 & 85,7 \\
\hline & Sim & - & - & 1 & 33,3 & 1 & 14,3 \\
\hline Diabetes & Não & 4 & 100 & 3 & 100 & 7 & 100 \\
\hline Doença mental & Não & 4 & 100 & 3 & 100 & 7 & 100 \\
\hline Outra & Não & 4 & 100 & 3 & 100 & 7 & 100 \\
\hline \multicolumn{8}{|l|}{ População especial } \\
\hline \multirow{2}{*}{ População privada de liberdade } & $\operatorname{sim}$ & - & - & 1 & 33,3 & 1 & 14,3 \\
\hline & Não & 4 & 100 & 2 & 66,7 & 6 & 85,7 \\
\hline \multirow{2}{*}{ Profissional de saúde } & $\operatorname{Sim}$ & - & - & 1 & 33,3 & 1 & 14,3 \\
\hline & Não & 4 & 100 & 2 & 66,7 & 6 & 85,7 \\
\hline Imigrantes & Não & 4 & 100 & 3 & 100 & 7 & 100 \\
\hline Total & & 4 & 57,1 & 3 & 42,9 & 7 & 100 \\
\hline
\end{tabular}

Quanto ao perfil clínico e do tratamento, a maioria dos casos foi notificada em 2016 (42,9\%), na referência terciária $(85,7 \%)$, caso novo ou reingresso após abandono (42,9\%), forma clínica pulmonar $(71,4 \%)$, radiografia de tórax suspeito para a TB $(85,7 \%)$, baciloscopia de escarro negativa (71,4\%), TMR-TB não realizado (57,1\%), cultura de escarro não realizada (57,1\%), teste de sensibilidade em branco (100\%), controle mensal em branco, com um a três contatos identificados $(42,9 \%)$ e nenhum examinado $(85,7 \%)$, tratamento na referência terciária $(57,1 \%)$, não realização do TDO (85,7\%), tempo de tratamento de 61 a 120 dias (28,6\%) e mais do que 181 dias (28,6\%), além do elevado percentual de abandono do tratamento (57,1\%) (Tabela 2). 
Tabela 2. Distribuição do perfil clínico e do tratamento das pessoas em situação de rua coinfectadas TB/HIV, quanto ao sexo feminino e masculino, em Porto Velho, Rondônia, no período de 2015 a 2018

\begin{tabular}{|c|c|c|c|c|c|c|c|c|}
\hline \multirow{2}{*}{\multicolumn{3}{|c|}{ Variáveis }} & \multicolumn{2}{|c|}{ Feminino } & \multicolumn{2}{|c|}{ Masculino } & \multicolumn{2}{|c|}{ Total } \\
\hline & & & $\mathbf{n}$ & $\%$ & $\mathbf{n}$ & $\%$ & $\mathbf{N}$ & $\%$ \\
\hline \multirow{3}{*}{ Ano da notificação } & \multicolumn{2}{|c|}{2015} & 2 & 50 & - & - & 2 & 28,6 \\
\hline & \multicolumn{2}{|c|}{2016} & 1 & 25 & 2 & 66,7 & 3 & 42,9 \\
\hline & \multicolumn{2}{|c|}{2018} & 1 & 25 & 1 & 33,3 & 2 & 28,6 \\
\hline \multirow{3}{*}{$\begin{array}{l}\text { Unidade de } \\
\text { notificação }\end{array}$} & \multicolumn{2}{|c|}{ Referência Terciária } & 4 & 100 & 2 & 66,7 & 6 & 85,7 \\
\hline & \multicolumn{2}{|c|}{ APS } & - & - & 1 & 33,3 & 1 & 14,3 \\
\hline & \multicolumn{2}{|c|}{ Caso novo } & 2 & 50 & 1 & 33,3 & 3 & 42,9 \\
\hline \multirow[t]{2}{*}{ Tipo de entrada } & \multicolumn{2}{|c|}{ Recidiva } & - & - & 1 & 33,3 & 1 & 14,3 \\
\hline & \multicolumn{2}{|c|}{ Reingresso após abandono } & 2 & 50 & 1 & 33,3 & 3 & 42,9 \\
\hline \multirow{3}{*}{ Forma clínica } & \multicolumn{2}{|c|}{ Pulmonar } & 3 & 75 & 2 & 66,7 & 5 & 71,4 \\
\hline & \multirow{2}{*}{\multicolumn{2}{|c|}{$\begin{array}{c}\text { Extrapulmonar } \\
\text { Pulmonar+Extrapulmonar }\end{array}$}} & 1 & 25 & - & - & 1 & 14,3 \\
\hline & & & - & - & 1 & 33,3 & 1 & 14,3 \\
\hline & Radiografia de & Suspeito de TB & 4 & 100 & 2 & 66,7 & 6 & 85,7 \\
\hline & tórax & Não realizado & - & - & 1 & 33,3 & 1 & 14,3 \\
\hline & & Negativa & 4 & 100 & 1 & 33,3 & 5 & 71,4 \\
\hline & Baciloscopia de & Positiva & - & - & 1 & 33,3 & 1 & 14,3 \\
\hline & escarro & Não realizada & - & - & 1 & 33,3 & 1 & 14,3 \\
\hline & & Não realizado & 2 & 50 & 2 & 66,7 & 4 & 57,1 \\
\hline Exames diagnósticos & TMR-TB & $\begin{array}{l}\text { Detectável sensível à } \\
\text { Rifampicina }\end{array}$ & - & - & 1 & 33,3 & 1 & 14,3 \\
\hline & & Não detectável & 2 & 50 & - & - & 2 & 28,6 \\
\hline & Cultura de & Negativo & 3 & 75 & - & - & 3 & 42,9 \\
\hline & escarro & Não realizado & 1 & 25 & 3 & 100 & 4 & 57,1 \\
\hline & $\begin{array}{c}\text { Teste de } \\
\text { sensibilidade }\end{array}$ & Em branco & 4 & 100 & 3 & 100 & 7 & 100 \\
\hline & & Negativo & - & - & 1 & 100 & 1 & 14,3 \\
\hline & $1^{\circ}$ mês & Não realizado & 1 & 25 & - & - & 1 & 14,3 \\
\hline & & Em branco & 3 & 75 & 2 & & 5 & 71,4 \\
\hline & & Não realizado & 1 & 25 & - & - & 1 & 14,3 \\
\hline & $2^{\circ}$ mes & Em branco & 3 & 75 & 3 & 100 & 6 & 85,7 \\
\hline & & Não realizado & 1 & 25 & - & - & 1 & 14,3 \\
\hline Controle Mensal & & Em branco & 3 & 75 & 3 & 100 & 6 & 85,7 \\
\hline & & Não realizado & 1 & 25 & - & - & 1 & 14,3 \\
\hline & $4^{\circ}$ mes & Em branco & 3 & 75 & 3 & 100 & 6 & 85,7 \\
\hline & & Não realizado & 1 & 25 & - & - & 1 & 14,3 \\
\hline & mes & Em branco & 3 & 75 & 3 & 100 & 6 & 85,7 \\
\hline & & Não realizado & 1 & 25 & - & - & 1 & 14,3 \\
\hline & $6^{\circ}$ mes & Em branco & 3 & 75 & 3 & 100 & 6 & 85,7 \\
\hline & Contatos & Zero & 2 & 50 & 2 & 66,7 & 4 & 57,1 \\
\hline & Identificados & De 1 a 3 & 2 & 50 & 1 & 33,3 & 3 & 42,9 \\
\hline Controle de contatos & Contatos & Zero & 3 & 75 & 3 & 100 & 6 & 85,7 \\
\hline & Examinados & Em branco & 1 & 25 & - & - & 1 & 14,3 \\
\hline & Referê & a Terciária & 3 & 75 & 1 & 33,3 & 4 & 57,1 \\
\hline de de & Ambulató & Especializado & 1 & 25 & 1 & 33,3 & 2 & 28,6 \\
\hline & & PS & - & - & 1 & 33,3 & 1 & 14,3 \\
\hline & & ̃̃o & 3 & 75 & 3 & 100 & 6 & 85,7 \\
\hline TDO & & ranco & 1 & 25 & - & - & 1 & 14,3 \\
\hline & & ro & 1 & 25 & - & - & 1 & 14,3 \\
\hline & & 60 & - & - & 1 & 33,3 & 1 & 14,3 \\
\hline Tempo de tratamento & & a 120 & 1 & 25 & 1 & 33,3 & 2 & 28,6 \\
\hline & & a 180 & - & - & 1 & 33,3 & 1 & 14,3 \\
\hline & Mais & que 181 & 2 & 50 & - & - & 2 & 28,6 \\
\hline & & ura & 2 & 50 & - & - & 2 & 28,6 \\
\hline Situaçao de & & dono & 1 & 25 & 3 & 100 & 4 & 57,1 \\
\hline rramento & Tra & erência & 1 & 25 & - & - & 1 & 14,3 \\
\hline
\end{tabular}

Fonte: SINAN, 2019

Sinal convencional utilizado: - Dado numérico igual a zero não resultante de arredondamento. 
Ressalta-se ainda que o tempo médio de tratamento, independentemente do sexo, foi de 116,7 dias $(\mathrm{dp}= \pm 80,75)$, sendo o mínimo de zero e máximo de 225 dias, o que corresponde aproximadamente a quatro meses de tratamento.

\section{Discussão}

A PSR está inserida em um contexto de invisibilidade social e naturalização de suas condições de vida, o que atrelado às iniquidades sociais e de saúde, implica diretamente na definição de políticas públicas, adoção de estratégias eficazes, além do real conhecimento sobre o seu quantitativo, o qual inclui em Porto Velho, por exemplo, imigrantes de diferentes países, tais como Haiti e Venezuela.

Tais aspectos, somados ainda com a dificuldade de acesso aos serviços de saúde, mesmo com a atuação do Consultório na Rua, interferem no acompanhamento dos casos, bem como nas ações de vigilância desde o rastreamento dos SR até o término do tratamento. Neste sentido, é importante considerar que a subnotificação dos casos de TB entre a PSR merece atenção, além do diagnóstico do HIV para detecção precoce e início imediato da terapêutica.

Partindo deste pressuposto, é relevante frisar que a taxa de incidência encontrada neste estudo reflete a vulnerabilidade à qual este grupo populacional está exposto, cujo risco de adoecimento por TB chega a ser 56 vezes maior quando comparado à população em geral, e entre as pessoas vivendo com HIV, 28 vezes maior ${ }^{4}$, ainda que a saúde se caracterize como um direito constitucional e que requer como ponto de discussão prioritário nas diferentes esferas de governo, de modo intersetorial, a fim de atender aos princípios e diretrizes do Sistema Único de Saúde (SUS).

Embora a incidência dos casos de coinfecção TB/ HIV entre as PSR do município de Porto Velho não represente a real magnitude de ambos os agravos nessa população ( 1,2 casos/100 habitantes), estudos apontam incidências que variam de 6,21 casos de TB/HIV/100 habitantes ${ }^{10}$, e até 122 casos somente de TB/100 mil PSR 11 .
Em relação ao perfil sociodemográfico, a literatura aponta que a TB e o HIV acometem mais o sexo masculino que também constitui, em sua maioria, a PSR ${ }^{12}$, diferentemente dos achados encontrados neste estudo, onde foi possível identificar que a notificação no cenário estudado ocorreu entre o sexo feminino, o que reforça a complexidade do cuidado com a saúde das mulheres em situação de rua ${ }^{6,13}$.

No que diz respeito à faixa etária, raça/ cor autodeclarada parda, baixa escolaridade, identifica-se a semeIhança com outros estudos que abordam sobre a população em geral que adoeceram por $\mathrm{TB}^{14,15}$, e que não diferem com o perfil encontrado no censo da PSR ${ }^{12} \mathrm{e}$ nem com os achados evidenciados neste estudo.

É importante considerar ainda que apresentar doenças e agravos associados, tais como alcoolismo e uso de drogas ilícitas, também constituem como um dos principais motivos para viver na rua ${ }^{12}$, ao mesmo tempo em que interferem na continuidade do tratamento ${ }^{16}$ de ambas as comorbidades, TB e HIV, no retardo na procura pelo serviço de saúde, uma vez que que o uso de psicoativos ocultam os sintomas e afetam a percepção acerca do próprio estado de saúde ${ }^{17}$. Isto influencia no agravamento dos sinais clínicos, elucidação diagnóstica e até mesmo continuidade do tratamento devido à complexidade no serviço de referência terciária, conforme também encontrado neste estudo.

Em conformidade a isso, um estudo realizado em Portugal evidenciou uma taxa de resultados desfavoráveis do tratamento da TB entre a PSR, significativamente, associada ao aumento da idade, uso de drogas injetáveis e coinfecção por HIV ${ }^{11}$, o que contribui para o entendimento da associação destes fatores de risco com os resultados identificados no presente estudo.

Além disso, 100\% das PSR com a coinfecção TB/HIV possuíam o diagnóstico de aids, o que questiona a efetividade das estratégias que vem sendo utilizadas para o acompanhamento e tratamento do HIV pelos serviços de referência integrados com o Consultório na Rua, considerando que se espera a perspectiva da supressão viral, na tentativa da melhoria da qualidade de vida e prognóstico de ambas as doenças, embora sejam conhecidos os demais fatores que influenciam na adesão e seguimento para essa população. 
Outro aspecto a ser considerado é a qualidade dos dados, uma vez que a mesma PSR com a coinfecção TB/HIV, também se caracterizava, concomitantemente, como privada de liberdade e profissional de saúde, conforme registro no SINAN. Nestes casos, a educação permanente em saúde entra como uma ferramenta em potencial para qualificação contínua dos profissionais de saúde quanto à correta notificação dos casos, a fim de evitar dubiedades. Entendese que a qualidade dos dados são ferramentas importantes para obter informações que contribuem para o planejamento de ações em saúde, bem como o despendimento de recursos necessários para levar à frente estratégias de prevenção de doenças, promoção da saúde e diminuição de agravos.

No perfil clínico, é possível questionar sobre a ausência de notificação desta PSR com a coinfecção TB/HIV no ano de 2017, considerando ainda que o tipo de entrada identificado como caso novo ou reingresso após abandono contribuem com a continuidade da cadeia de transmissão, principalmente pela forma pulmonar e desfechos desfavoráveis, os quais interferem no aumento da prevalência da doença no município.

A elucidação diagnóstica foi realizada com o auxílio de exames de imagem, como o raio-x, por sua fácil realização, e os laboratoriais, como a baciloscopia de escarro, na qual, embora neste estudo tenham obtido resultado negativo, destaca-se a não realização de outros exames laboratoriais, tais como o TMR-TB para os casos novos, com o intuito de agilizar o resultado para o diagnóstico, a cultura de escarro, uma vez que existe elevados percentuais de MNT e reingresso após abandono no estado de Rondônia, além do teste de sensibilidade para detecção de resistência aos fármacos e a efetividade destes antituberculostáticos por meio da baciloscopia de escarro para controle mensal.

Estudos vêm evidenciando o impacto da oferta de triagens direcionadas e oportunas, por meio de exames de raio-x para a PSR e usuários de drogas ilícitas, como uma nova abordagem de rastreamento e busca ativa de TB, que poderia ser integrada às ações de controle da doença por meio dos Programas de Controle da TB, por propiciar uma alta taxa de detecção de $\operatorname{casos}^{18,19}$, considerando especialmente regiões de baixa incidên$\mathrm{cia}^{20}$, como identificada no município.
Dentre as ações de vigilância e controle da TB, ressalta-se que o controle de contatos permite identificar os casos de Infecção latente pelo Mycobacterium tuberculosis (ILTB) e TB ativa, bem como a oferta e realização do TDO para garantir a adesão ao tratamento, fortalecer o vínculo e aproximar serviço, usuário, família e comunidade, principalmente para aqueles que possuem e estão inseridos em contextos de vulnerabilidade social, tal como a PSR que possui ainda a coinfecção TB/HIV enquanto doenças estigmatizantes e que potencializam a exclusão social.

Como limitações deste estudo, ressalta-se as fragilidades na retroalimentação e completude dos dados no SINAN, uma vez que o não registro das informações sobre as ações realizadas não permite inferir a sua realização (ou não), bem como duplicidade de atividades, além de não contribuir com o planejamento e não permitir conhecer a real magnitude do problema entre a PSR coinfectada pela TB e HIV. De modo complementar, dados oficiais e atualizados no município, ausência de pesquisas e estudos sobre esta população.

\section{Conclusão}

Diante dos achados neste estudo, verificou-se que o perfil sociodemográfico incluiu principalmente o sexo feminino, faixa etária entre 31 a 38 anos, raça/ cor autodeclarada parda, baixa escolaridade, doenças e agravos associados, tais como aids, alcoolismo e uso de drogas ilícitas, além de não se caracterizarem como população especial. De modo complementar, foram notificados e tratados na referência terciária, eram caso novo ou reingresso após abandono, com a forma clínica pulmonar, radiografia de tórax suspeito para a TB, baciloscopia de escarro negativa, TMR-TB e cultura de escarro não realizados, teste de sensibilidade e controle mensal em branco, com um a três contatos identificados e nenhum examinado, TDO não realizado, tempo de tratamento de 61 a 120 dias e mais do que 181 dias, além do elevado percentual de abandono do tratamento.

Do ponto de vista social, este estudo possui implicações para a saúde pública que perpassam o enfoque das iniquidades e dos determinantes sociais de saúde, uma vez que a PSR possui demandas específicas, as quais devem ser consideradas, especialmente em relação ao acesso aos serviços e ações de saúde ofertadas. 
A notificação e tratamento dos casos na referência terciária, e o perfil clínico de risco contribuem para a manutenção da cadeia de transmissão entre a PSR e a população geral, agravamento dos sinais e sintomas, e pode ter refletido no desfecho desfavorável do abandono. Neste sentido, questiona-se a efetividade das ações de controle da doença na APS e a articulação com o consultório na rua e demais níveis de atenção na Rede de Atenção à Saúde.

\section{Agradecimentos}

Agradecemos ao Grupo de Estudos em Tuberculose (GET) da Universidade Federal de Rondônia, certificado pelo diretório do Conselho Nacional de Desenvolvimento Científico e Tecnológico (CNPq).

\section{Contribuições das autoras}

Orfão NH e Silva KM participaram da concepção, delineamento, busca e análise estatística dos dados da pesquisa, interpretação dos resultados e redação do artigo científico. Ferreira MRL participou da redação e revisão crítica do artigo científico. Brunello MEF participou da revisão crítica do artigo científico. Todos as autoras aprovam a versão final a ser publicada e estão de acordo por todos os aspectos do trabalho, garantindo sua precisão e integridade.

\section{Conflitos de interesses}

Nenhum conflito financeiro, legal ou político envolvendo terceiros (governo, empresas e fundações privadas, etc.) foi declarado para nenhum aspecto do trabalho submetido (incluindo, mas não se limitando a subvenções e financiamentos, participação em conselho consultivo, desenho de estudo, preparação de manuscrito, análise estatística, etc.).

\section{Referências}

1. World Health Organization. Global tuberculosis report 2019 [Internet]. Geneva: WHO; 2019 [citado em 2020 out 20]. Disponível em: https://apps.who.int/iris/bitstream/hand le/10665/336069/9789240013131-eng.pdf?ua=1

2. Ministério da Saúde (Brasil), Secretaria de Vigilância em Saúde. Boletim Epidemiológico: Brasil Livre da Tuberculose: evolução dos cenários epidemiológicos e operacionais da doença [Internet]. Brasília: Ministério da Saúde/Secretaria de vigilância em Saúde. 2019. Disponível em: https://portalarquivos2.saude.gov.br/ images/pdf/2019/marco/22/2019-009.pdf
3. Ministério da Saúde (Brasil), Secretaria de Vigilância em Saúde. Boletim Epidemiológico de Tuberculose 2020 [Internet]. Brasília: Ministério da Saúde/Secretaria de Vigilância de Saúde. 2020. Disponível em: http://www.aids.gov.br/pt-br/pub/2020/boletimepidemiologico-de-turbeculose-2020

4. Ministério da Saúde (Brasil), Secretaria de Vigilância em Saúde. Manual de recomendações para o controle da tuberculose no Brasil [Internet]. Brasília: Ministério da Saúde/Secretaria de Vigilância em Saúde. 2019. Disponível em: https://bvsms.saude. gov.br/bvs/publicacoes/manual_recomendacoes_controle_ tuberculose_brasil_2_ed.pdf

5. Silva RP, Leão VAS, Santos ESV, Costa GN, Santos RV, Carvalho VT, et al. Assistência de Enfermagem a pessoa em situação de rua. Revista Recien [Internet]. 2017;7(20):31-9. Disponível em: https://www.recien.com.br/index.php/recien/ article/view/222\#: :text=Aponta\%2Dse\%20que\%20a\%20 enfermagem, melhoria\%20da\%20qualidade $\% 20$ de $\% 20$ vida

6. Alecrim TFA, Mitano F, Reis AA, Roos CM, Palha PF, ProttiZanatta ST. Experiência dos profissionais de saúde no cuidado da pessoa com tuberculose em situação de rua. Rev. esc. enferm. USP. 2016;50(5):808-15. http://dx.doi.org/10.1590/s0080$\underline{623420160000600014}$

7. Instituto Brasileiro de Geografia e Estatística. População [Internet]. 2019. [citado em 2019 mai 31]. Disponível em: https:// cidades.ibge.gov.br/brasil/ro/portovelho/panorama

8. Ministério da Saúde (Brasil). Cadastro Nacional de Estabelecimentos de Saúde (CNES) [Internet]. 2020. [citado em 2019 mai 31]. Disponível em: http://cnes.saude.gov.br/pages/ estabelecimentos/consulta.jsp

9. Resolução 466, de 12 de dezembro de 2012 (Brasil). Aprova as diretrizes e normas regulamentadoras de pesquisas envolvendo seres humanos e revoga as Resoluções CNS nos. 196/96,

303/2000 e 404/2008 [Internet]. Diário Oficial da União. 2012 dez 12. Disponível em: https://bvsms.saude.gov.br/bvs/saudelegis/ cns/2013/res0466_12_12_2012.html

10. Davy-Mendez T, Shiau R, Okada RC, Moss NJ, Huang S, Murgai $\mathrm{N}$, et al. Combining surveillance systems to investigate local trends in tuberculosis-HIV co-infection. AIDS Care. 2019;31(10):1311-8. http://dx.doi.org/10.1080/09540121.2019.1576845

11. Dias M, Gaio R, Sousa P, Abranches M, Gomes M, Oliveira O, et al. Tuberculosis among the homeless: should we change the strategy?. Int. J. Tuberc. Lung. Dis. 2017;21(3):327-32. http://dx.doi. org/10.5588/ijtld.16.0597

12. Cortizo RM. População em situação de rua no Brasil: o que os dados revelam? [Internet]. Brasília: Ministério da Cidadania; 2019. Disponível em: https://aplicacoes.mds.gov.br/sagirmps/ ferramentas/docs/Monitoramento_SAGI_Populacao_situacao_rua. pdf 
13. Rossetto M, Maffacciolli R, Rocha CMF, Oliveira DLLC, Serrante L. Coinfecção tuberculose/HIV/aids em Porto Alegre, RS - invisibilidade e silenciamento dos grupos mais afetados. Rev. Gaúcha Enferm. 2019;40:e20180033. http://dx.doi. org/10.1590/1983-1447.2019.20180033

14. Silva LT, Felipini MCC, Oliveira TB, Brunello MEF, Orfão NH. Perfil epidemiológico da tuberculose no serviço de referência do estado de Rondônia. Rev. Epidemiol. Controle Infecç. 2019;9(1):4854. http://dx.doi.org/10.17058/reci.v9i1.12249

15. Siqueira TC, Bonfim RO, Ferreira MRL, Orfão NH. Mortalidade entre os portadores de tuberculose em Porto Velho (RO). Saúde e Pesquisa [Internet]. 2018;11(3):441-50. Disponível em: https:// periodicos.unicesumar.edu.br/index.php/saudpesq/article/ view/6753

16. Ferreira MRL, Bonfim RO, Siqueira TC, Orfão NH. Abandono do tratamento da tuberculose: uma revisão integrativa. Rev. Enferm. Contemp. 2018;7(1):63-71. http://dx.doi.org/10.17267/23173378rec.v7i1.1579
17. Zuim RCB, Trajman A. Itinerário terapêutico de doentes com tuberculose vivendo em situação de rua no Rio de Janeiro. Physis. 2018;28(2):e280205. http://dx.doi.org/10.1590/s0103$\underline{73312018280205}$

18. Goetsch U, Bellinger OK, Buettel KL, Gottschalk R. Tuberculosis among drug users and homeless persons: impact of voluntary X-ray investigation on active case finding. Infection. 2012;40(4):389-95. http://dx.doi.org/10.1007/s15010-011-0238-x

19. Vries G, Van Hest RA, Richardus JH. Impact of mobile radiographic screening on tuberculosis among drug users and homeless persons. Am. J. Respir. Crit. Care Med. 2007;176(2):2017. http://dx.doi.org/10.1164/rccm.200612-18770C

20. Paquette K, Cheng MP, Kadatz MJ, Cook VJ, Chen W, Johnston JC. Chest radiography for active tuberculosis case finding in the homeless: a systematic review and meta-analysis. Int. J. Tuberc. Lung Dis. 2014;18(10):1231-6. http://dx.doi.org/10.5588/ ijtld.14.0105 